\title{
Universality caused: the case of renormalization group explanation
}

\section{Emily Sullivan ${ }^{1}$}

Received: 31 May 2018 / Accepted: 13 June 2019 / Published online: 29 June 2019

(C) The Author(s) 2019

\begin{abstract}
Recently, many have argued that there are certain kinds of abstract mathematical explanations that are noncausal. In particular, the irrelevancy approach suggests that abstracting away irrelevant causal details can leave us with a noncausal explanation. In this paper, I argue that the common example of Renormalization Group (RG) explanations of universality used to motivate the irrelevancy approach deserves more critical attention. I argue that the reasons given by those who hold up RG as noncausal do not stand up to critical scrutiny. As a result, the irrelevancy approach and the line between casual and noncausal explanation deserves more scrutiny.
\end{abstract}

Keywords Causal explanation · Non-causal explanation · Universality $\cdot$ Renormalization group $\cdot$ Critical phenomena $\cdot$ Abstraction

\section{Introduction}

Many philosophers argue that there are certain kinds of abstract mathematical explanations that are noncausal (Batterman 2000, 2010; Colyvan 2001; Felline 2018; Jansson and Saatsi 2017; Lange 2013; Lipton 2004; Pincock 2015; Reutlinger 2014; Rice 2015). Their arguments have been quite persuasive and there is a growing number of philosophers of science who are either pluralists about explanation or seeking alternative theories of explanation that can unify both noncausal and causal explanations. Proponents of noncausal explanation utilize different approaches to make their case and each approach trades on different scientific examples. ${ }^{1}$ For instance, one such approach is to argue that there are scientific explanations that explain why something is necessary through mathematics or graph theory in a way that fails to be causal (Lange 2013, 2017). Another approach is to argue that abstracting away irrelevant causal

${ }^{1}$ For a recent overview of noncausal approaches to explanation see Reutlinger (2017).

Emily Sullivan

e.e.sullivan-mumm@tudelft.nl

1 Delft University of Technology, Delft, Netherlands 
details to find commonality between disparate systems can leave us with a noncausal explanation (Batterman 2000, 2010; Reutlinger 2014). Call this the irrelevancy approach.

In this paper, I argue that the common example of Renormalization Group (RG) explanations of universality used to motivate the irrelevancy approach deserves more critical attention. I argue that the reasons given by those who hold up RG as noncausal do not stand up to critical scrutiny, suggesting that the consensus treating RG explanations as noncausal is premature. As a result, the irrelevancy approach and the line between causal explanation and noncausal explanation deserves more attention.

In what follows, I first discuss the distinguishing features of noncausal explanation (§2). From there, I turn to the case at hand-RG explanations of universality (§3). In $\S 4$, I introduce the noncausal interpretation of RG. In $\S 5$, I argue that the reasons given to motivate a noncausal interpretation fall away under scrutiny, thus placing the burden of proof back on those who wish to argue that RG explanations of universality is indeed an instance of noncausal explanation. I conclude with a brief discussion of the broader implications for noncausal accounts of explanation.

\section{Causes and noncausal explanation}

Part of the complexity in the debate surrounding causal and noncausal explanation is that many are working under different theories of explanation, so in order to make headway a few clarifications are in order.

First, it is important to take note of what is at stake in the debate surrounding noncausal and causal explanation. Ultimately, the issue is whether we have a pressing challenge to causal-only approaches to scientific explanation. The threat comes when the principal way of explaining an event involving physical phenomena relies on a relation other than a causal one. While there are fruitful discussions surrounding the nature of mathematical explanation and how this can be unified with explanations in the physical sciences, we should not expect a causal account of explanation to be able to cover cases in theoretical mathematics. As such, the types of cases introduced by noncausalists engaged in the critique of causal explanatory theories underscore not simply that there exists a mathematical dependency, but that this dependency helps to explain an event involving physical phenomena.

Second, my aim is to stay fairly neutral on what a cause itself is, and what the correct approach to explanation is. Following Reutlinger (2014, 2016), I will adopt the following commonsense notion of a cause: a cause involves an asymmetrical dependence relation between events or types of events. A cause happens before its effect and the effect cannot explain the cause. Adopting this notion comes with a few advantages. First, it allows me to argue on Reutlinger's (2014) own terms when it comes to the case of $\mathrm{RG}$, while still leaving it fairly open for compatibility with other theories of causation. Second, it makes my job fairly demanding. For example, this notion of causation is more restrictive than Skow's $(2014,2016)$ account of causal information. And it is at least as demanding as Woodward's (2003) interventionist account, which some critics see as making room for more causes than Reutlinger's "common sense" view (Saatsi and Pexton 2013). 
I will draw on several insights from various accounts of causal explanation (e.g. Potochnik 2015; Strevens 2008; Woodward 2003). My aim is not to argue for a particular causal interpretation of RG explanations of universality. My aim is to shift the burden of proof back onto the noncausalists by showing that the reasons on offer $d o$ not prevent a causal interpretation.

Lastly, since my interlocutors argue that RG is noncausal by employing working definitions of noncausal explanation, I frame the discussion in terms of what it would take for an explanation to count as noncausal as opposed to causal. ${ }^{2}$

As a starting point, Reutlinger (2014), in his paper on RG explanations of universality, defines a noncausal explanation (which I am calling $\mathrm{NC}$ ) as follows:

NC: An explanation is noncausal iff the explanans contains at least one noncausal element $e$, and $e$ ensures the success of the explanation. (p. 1165)

$\mathrm{NC}$ as it stands is vague and needs more clarification. If 'ensures' simply picks out a necessary condition for explanation, then $\mathrm{NC}$ is too exclusionary. It means that any set of explanantia which includes at least one noncausal piece of information thereby qualifies the explanation as noncausal. As a consequence, several explanations that many take to be causal are actually noncausal after all. For example, any explanation that has boundary conditions or basing generalizations would be considered noncausal. This is because boundary conditions are necessary for the entailment of the explanandum and yet may not provide any causal information. A familiar example here is that in order to explain why all ravens are black we not only need to provide a causal story that includes facts about genetics and biology, but also include a fact along the lines, "all ravens have some property $p$." Yet simply having a property is not by itself causal information. ${ }^{3}$

On a more charitable reading, we can discount such conditions as candidates for ruling out an explanation as causal by making the following modification to NC:

$\mathrm{NC}^{*}$ : An explanation is noncausal iff the explanans contains at least one noncausal element $e$, and e primarily ensures the success of the explanation.

With this change, in order for an explanation to be noncausal there must be a noncausal element in the explanation that is not only necessary for the success of the explanation, but which is also a primary reason why the explanation succeeds. Consider a paradigm example of noncausal explanation of an event through mathematical necessity. Why couldn't Sally's dad divvy up the 23 strawberries to her and her two brothers evenly without cutting them? The best explanation seems to be because 23 cannot be divided by 3 evenly. ${ }^{4}$ It is necessary for the success of this explanation that there are 23

\footnotetext{
${ }^{2}$ My arguments are also not directed toward the monism project in general. Monists seek to develop a theory of explanation that can equally account for both causal and noncausal explanations (see e.g. Povich 2016). Since RG explanations are explanations, what I say here can largely be made compatible with compelling monist theories of explanation. The challenge I am raising is that it is not as obvious as it is now assumed that RG explanations of universality fall on the noncausal side.

${ }^{3}$ For a more detailed discussion of basing generalizations see Strevens (2008, 228-265).

${ }^{4}$ This example is from Lange (2013).
} 
strawberries; however, the salient explanatory feature here is not only the background condition of how many strawberries there are, but that 23 is not divided by 3 evenly. ${ }^{5}$ According to $\mathrm{NC}^{*}$, the reason that this strawberry case is noncausal is not because it provides noncausal information regarding the number of strawberries; it is noncausal because a mathematical fact about division is the primary reason the explanation succeeds.

However, $\mathrm{NC}^{*}$ is still underspecified. Often causal generalizations and laws are expressed mathematically, such as probabilistic causal laws and Newtonian laws of motion. Further, in many explanations, mathematical manipulation of these laws figures in how the explanandum is explained. In view of $\mathrm{NC}^{*}$, any explanation of physical phenomena that uses mathematics as a representative tool is not a causal explanation because the mathematical facts and operations are playing a primary role in the success of the explanation. However, an explanation that includes a mathematical derivation should not automatically rule out the explanation as causal. Causal information can be expressed mathematically, and Reutlinger himself does not want to exclude such cases. He gives the example that one can explain the event of a billiard ball colliding with another with the use of a causal regularity expressed in mathematical language, such as Newton's laws of motion, and computing the impact of the collision by citing the particular velocity of the billiard ball $(2014,1165)$. In this case, the mathematical representation expresses causal information that holds onto our commonsense notion of causation. There is an asymmetrical dependence relation between the force behind the ball that strikes the other, and the effect cannot explain the cause. It should not matter how the causal relation is expressed when determining whether the explanation is causal, but whether it represents causal information in the explanation.

Furthermore, it might be that the laws themselves are noncausal. Russellians, for example, hold the view that many (if not all) fundamental physical laws are noncausal laws because they express a symmetrical dependence (cf. Russell 1913). ${ }^{6}$ And since causes are fundamentally asymmetrical, the laws cannot be causal laws. However, this view is far from uncontroversial. And even if we endorse such a view, it does not thereby preclude that such a law can causally explain. For example, neo-Russellians seek to maintain causal dependence while holding on to Russellian intuitions regarding symmetry at the fundamental level (e.g. Albert 2000; Loewer 2007). Indeed, examples of symmetrical laws include the law of gravity and Newton's law of motion referred to in the billiard ball case (cf. Farr and Reutlinger 2013). Yet, again, many share the belief that it is possible to causally explain using the law of gravity and Newton's laws of motion when discussing events such as apples falling from trees, or billiard balls colliding.

For the purposes of this paper, it does not matter so much what the right story is for how symmetrical laws can maintain causal dependence in explanation; it only matters that such a story is available. Moreover, noncausalists should not find comfort in only relying on fundamental symmetrical laws to make their case for noncausal explanation. It is a large methodological burden to require a version of Russellian causaleliminativism for the case for noncausal explanation to get off the ground. Just as

\footnotetext{
${ }^{5} \mathrm{I}$ am leaving the notion of salience and a primary reason imprecise. So, while also a bit vague, it stresses the explanatory role more so than NC. Any conclusions that I draw based on this, I take it, will be uncontroversial. For an interesting discussion on incidental counterfactuals and explanatory power see Khalifa et al. (2018).

${ }^{6}$ For an overview on causal asymmetry in physics, including approaches to maintain time-asymmetry at the fundamental level, see Blanchard (2016) and Farr and Reutlinger (2013).
} 
noncausalists are wary of those claiming that all scientific explanations are causal, causalists should be wary of noncausal explanatory views that entail all explanations involving laws are noncausal. It's clear that a third modification to our account of noncausal explanation is needed:

NC**: An explanation is noncausal iff the explanans contains at least one noncausal element $e$, $e$ primarily ensures the success of the explanation, and $e$ does not have a causal-tether.

According to $\mathrm{NC}^{* *}$, mathematical representations and derivations do not automatically make an explanation noncausal. Insofar as the mathematical representations have a causal-tether to the phenomenon, they can still causally explain, as seen in the billiard ball case and the gravity case.

So, are there explanations that satisfy $\mathrm{NC}^{* *}$ ? This of course is a matter of dispute. Since it is not my aim in the paper to argue that all scientific explanations are causal, we can, for the sake of argument, grant that the strawberry example described above satisfies $\mathrm{NC}^{* *}$. The intuition of pluralists and critics of causal theories of explanation is that the strawberry case is noncausal because the fact that 3 does not divide evenly into 23 does not represent any causal information about divvying up strawberries. There is no time-asymmetry involved in the mathematical operation of division. Mathematical operations are not events. There is nothing about the physical structure of strawberries that prevents them from being divided in this way. Furthermore, the explanation is independent from the actual laws of nature (Jansson and Saatsi 2017). The physical laws in the world could be different, and the explanation would still hold.

On the other hand, there is something about the constitution of billiard balls that determines how they will behave under specific conditions regarding force and momentum, thus ensuring that a causal-tether remains. There is a clear time-asymmetry, and the explanation depends on the physical laws of nature. According to Reutlinger's counterfactual theory of explanation, the billiard ball case is explanatory by virtue of exhibiting a causal counterfactual dependency along the lines of: "if ball A had been struck with less force, then ball A would not have collided with ball B." The strawberry case is explanatory by virtue of exhibiting a noncausal counterfactual dependency along the lines of: "if the strawberries numbered 33, then they would have been able to have been split up evenly."

Whatever we should think of the strawberry case, what I argue here is that in the case of RG explanations of universality, where the noncausal interpretations are not chiefly rooted in mathematical necessity, but stem from the irrelevancy approach, the story for why RG representations do not have a causal-tether remains under-motivated.

Let us now move to the case at issue: renormalization group explanations of universality and continuous phase transitions.

\section{The case: Universal macrobehavior of phase transitions}

Consider a kettle of water heating up. At a certain point the water will undergo a transition from a liquid to a gas. At a basic level, one can get a grip on this phenomenon 
by understanding the amount of heat needed for a transition to occur. Specifically, as one learns in an introductory physics class, one can calculate the heat absorbed by multiplying the mass by the heat of vaporization for the substance in question. This is a generalization about how much heat is absorbed when a substance undergoes a firstorder phase transition. It is also an abstraction. It ignores the details. It does not mention microscopic particularities of the substance in question and can be applied to systems other than water.

We also find strikingly similar macrobehavior among very diverse systems undergoing a continuous phase transition (also called a second-order phase transition), such as magnetic systems and other various fluids. In the previous example of boiling water, we have a first-order phase transition because it involves non-zero latent heat and a discontinuity in density at the critical temperature. However, in a continuous phase transition, properties like density or magnetic moment change continuously. It is the second derivative, such as compressibility or magnetic susceptibility, that becomes discontinuous. ${ }^{7}$

A paradigm case of a continuous phase transition is the transition in magnetic systems between paramagnetic and ferromagnetic states. In these systems, the net magnetization changes when they are cooled or heated. In instances of high heat, the system's spins are disordered and point in random directions, leaving no net magnetization. As the system is cooled and reaches its critical temperature, the spins align and become ordered, making the system regain magnetization. ${ }^{8}$ As such, magnetization is the system's order parameter. An order parameter is a physical quantity (often the thermal average) that is zero on one side of the transition and moves away from zero on the other side. Generally speaking, as the temperature increases, the order parameter approaches zero and the system becomes more disordered.

Different systems have different order parameters. ${ }^{9}$ For example, in the case of water and other liquid-gas transitions, the order parameter is density, with density change approaching zero during a continuous phase transition. There is no general approach to defining a system's order parameter(s). As Binney et al. explain:

[O]ne has to consider each new physical system afresh. We can best indicate what sort of thing an order parameter is by giving a number of examples, starting with the phase transitions with which we are already familiar. $(1992,13)$

The interesting thing is that despite the different order parameters of systems and other microphysical details of each, disparate systems display similar macrobehavior at the critical point, and thus share the same universality class. The universality class is used to characterize the system such that we can predict how it will behave, determine which other systems have similar macrobehavior, and group these systems together in a meaningful way. We observe that several diverse systems that have different microscopic properties share the same long-range behavior, have a similar underlying order, share the same critical exponents, and collapse onto a common coexistence curve. The

\footnotetext{
7 There are special cases where water can undergo a continuous phase transition, such as steadily increasing pressure and temperature inside a stout vessel (Binney et al. 1992, 3).

${ }^{8}$ In this example, particles are ordered in respect to their spatial position. In other cases, this is done with respect to their momentum (e.g. superfluidity of liquid helium and superconductivity).

${ }^{9}$ Note that some complex systems can have more than one order parameter.
} 
difficult question, however, is why? Renormalization group (RG) methods provide us with an answer. ${ }^{10}$

We start by observing that when a system is far away from its critical point, the interactions of the particles in the system are confined to those nearby, exhibiting small scale fluctuations. The correlation length, $\xi$, is a spatial correlation of the system that characterizes the range of fluctuations of a variable (or "degree of freedom") within the system. The correlation length is a function of temperature, $\xi(T)$. In the case of a fluid, the larger the $\xi(T)$, the more fluctuations of density that occur in one area of the fluid will be correlated with fluctuations in another area at a large distance. "[A]t a critical point the shortrange inter-molecular forces contrive to generate large dynamical structures," uncovering interesting macro-level behavior (Binney et al. 1992, 20). Importantly, a system undergoing a continuous phase transition has a correlation length of infinity at the critical temperature, $T_{c}$. This relationship can be expressed as follows:

$$
\xi(T) \rightarrow \infty \text { as } T \rightarrow T_{c}
$$

Due to the divergence of the correlation length at the critical point, the behavior of a system at its critical point becomes intractable. Batterman $(2000,12)$ sums up the point nicely:

[C]orrelations at every length scale (between near as well as extremely distant components) contribute to the physics of the system as it undergoes a phase transition. This is a highly singular mathematical problem and is, in effect, completely intractable. It is relatively easy to deal with correlations that obtain between pairs of particles, but when one must consider correlations between three or more - even more than $10^{23}$ - particles, the situation is hopeless.

As a solution, RG methods were developed to restrict the degrees of freedom and in the process highlight relevant couplings and dispense with irrelevant couplings so that one can understand the system's behavior at the critical point and the universality of critical phenomena. This is done through a coarse-graining procedure that 'zooms out' from the short-range interactions in the system to reveal longer and longer-distance interactions. The relevant couplings are those that grow larger as the length scale increases, and the irrelevant couplings, (ultimately the microphysical couplings) decrease and wash out under renormalization group transformations. There are many sophisticated ways of modeling and executing this coarse-graining procedure. In what follows, I describe a relatively simple method developed by Leo P. Kadanoff (1966). ${ }^{11}$

\footnotetext{
${ }^{10}$ Since my ultimate goal in this paper is to make a philosophical point about the causal nature of RG explanations, I will present a mostly non-technical treatment of RG and point to resources along the way that an interested reader can look to in order to delve into the nitty-gritty of RG methods. As a start see Batterman (2000) and Morrison's (2014) treatment of RG, which remains on the less technical side. For the more ambitious reader, see Butterfield (2011, 2014), Butterfield and Bouatta (2012), and Kadanoff (2013) for a detailed description of the math behind RG as applied to continuous phase transitions and quantum field theory more generally.

${ }^{11}$ Kadanoff's block spin method is associated with real-space RG methods, where the techniques involve quantities dependent on position. Other methods, such as k-space (or momentum-space) involve Fouriertransformed quantities and are thought to be more generally applicable compared to real-space methods. See Binney et al. (1992) for more about the physics behind each, and Franklin (2018) for a discussion on the explanatory potential of each method. I take it that the exact coarse-graining implementation does not change the causal nature of critical phenomena explanations, so long as the original Hamiltonian represents the system(s) in question.
} 
The RG transformations are done on the system's Hamiltonian. A Hamiltonian is a function that tracks the energy of the system. Generally speaking, a Hamiltonian function represents the kinetic and potential energy of a system. More particularly, it focuses on the phase space of a system and maps the interactions between the degrees of freedom, spins, the effects of external fields, and so on. Using the Ising Model, the Hamiltonian for a ferromagnet is written as follows:

$$
\mathcal{H}=-J \sum_{i, j} \sigma_{i} \sigma_{j}-\mu H \sum_{i} \sigma_{i}
$$

where $H$ is the external field, $J$ is the coupling constant, and $\sigma_{i} \sigma_{j}$ are spin operators.

RG transformations iterate over the system's Hamiltonian resulting in a reduction in resolution. The original spin variables, which are defined with respect to some spatial direction, are replaced with new variables with each new transformation. The method known as the block spin method, shown in Fig. 1, depicts the iterative process. The average spin is taken between nearby neighbors to provide new spin variables representing a larger block. The new spin variables take the place of the old variables. This process is repeated again and again resulting in the distance between the sites becoming larger. ${ }^{12}$

Crucially, each new Hamiltonian function still tracks a system that displays similar behavior. With each new iteration, the Hamiltonian represents the same behavior of the system, but on a larger and larger scale. If the new Hamiltonian cannot account for the behavior we are trying to understand, then we begin to depart from the relevant phenomena. RG methods abstract away irrelevant microlevel interactions and shortrange behavior, representing long-range interactions and behavior on the macrolevel. ${ }^{13}$

The renormalization process is repeated until a fixed point is found, that is, a point at which no further iterations on the Hamiltonian show a change in correlation length or in the value of parameters. ${ }^{14}$ At the fixed point, the system's behavior is invariant to scale changes, giving rise to a renormalizable Hamiltonian. The only features that are relevant for the behavior of the system at the fixed point are those represented in the Hamiltonian. The system's Hamiltonian, or more precisely the coupling constants, are said to flow toward its fixed point. Kadanoff explains that "the act of renormalization is a sort of focusing in which many different irrelevant couplings fade away and we end up with a single fixed point representing a whole multi-dimensional continuum of different possible Hamiltonians" $(2013,34)$. He says further that "the fixed point concept describes a connection between the microscopic properties of the material, i.e. the interactions among its constituent particles and felids, and the behavior of the material on a conceptually infinite length scale. This connection is surprising and quite beautiful" $(2013,35)$.

The last step in the RG process is to utilize the fixed points to determine the value of the system's critical exponents, which are used to determine the universality class the system is in. Critical exponents describe behavior of various thermodynamic quantities near the critical point. For example, following Butterfield and Boutta (2012, 393-4), if

\footnotetext{
${ }^{12}$ Look to Kadanoff $(2013, \S 4)$ for a detailed discussion of the math behind RG.

13 This type of renormalization is a flow toward the infrared. There are other RG set-ups where there is not a coarse-graining, but a fine-graining procedure where the length-scale decreases. In this latter case it is a flow toward ultra-violet. For the sake of simplicity, I will continue to develop the case study for a flow toward the infrared. I take it that my main point of the causal nature of RG applies equally to both kinds of flow.

${ }^{14}$ Strictly speaking, this process would need to repeat infinitely to arrive at the fixed point.
} 


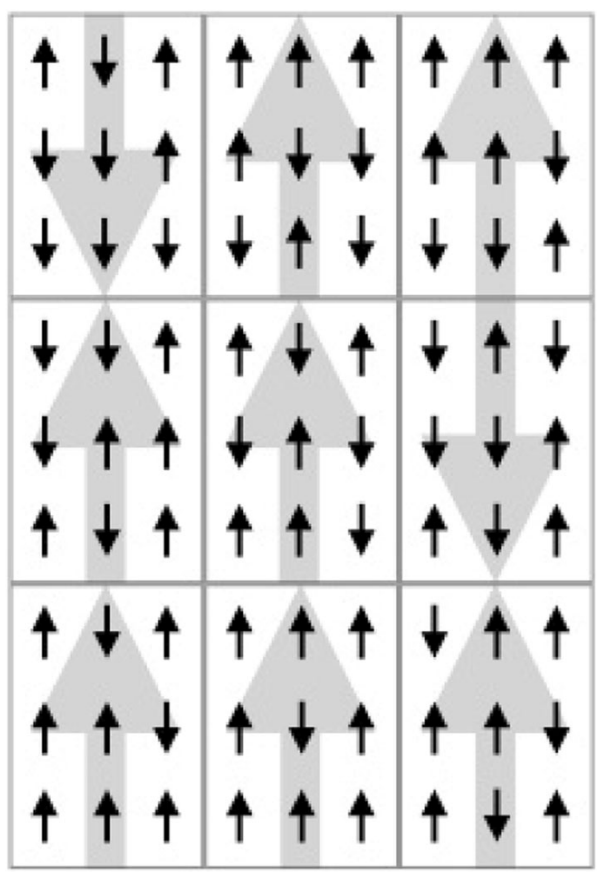

Fig. 1 Block Spin Illustration. Spins are broken up into blocks and averaged, leaving behind new spin variables

we express the reduced temperature as $\tau=\left(T-T_{c}\right) / T_{c}$ then we write the functions of correlation $\xi$, heat capacity $\mathcal{C}$, and isothermal susceptibility $\mathcal{X}$ as:

$$
\xi \sim|\tau|^{-v} \mathcal{C} \sim|\tau|^{-\alpha} \quad \mathcal{X} \sim|\tau|^{-\gamma}
$$

where, $\nu, \alpha$, and $\gamma$ are the critical exponents. The value of the critical exponents is not a function of the detailed microstructure of the system, but is determined by long-range fluctuations. Moreover, the critical exponents of each system within the same universality class share the same numerical value. In particular, using a model, like the above Ising model, standing in for different members within a universality class, we can see that disparate systems would flow to the very same fixed point, and thus share the same critical exponents.

Figure 2 shows the flow of Hamiltonians of disparate systems to the same fixed point. This means that disparate systems within a single universality class (e.g. fluids and ferromagnets) have the same relationship between their order parameters, degrees of freedom, and so on. In particular, it is found that each universality class depends on the spatial dimension, spin dimensionality, and on the symmetry properties of the system's order parameter (Batterman 2000, 127).

To sum up, the explanation of universal macrobehavior of continuous phase transitions through RG is crudely something like the following: the flow of Hamiltonians of each system within a universality class terminates at the same fixed point. At this point the irrelevant couplings of the system have been iterated out-leaving behind the largest length scale. At this point an infinite number of particles and infinite correlation 


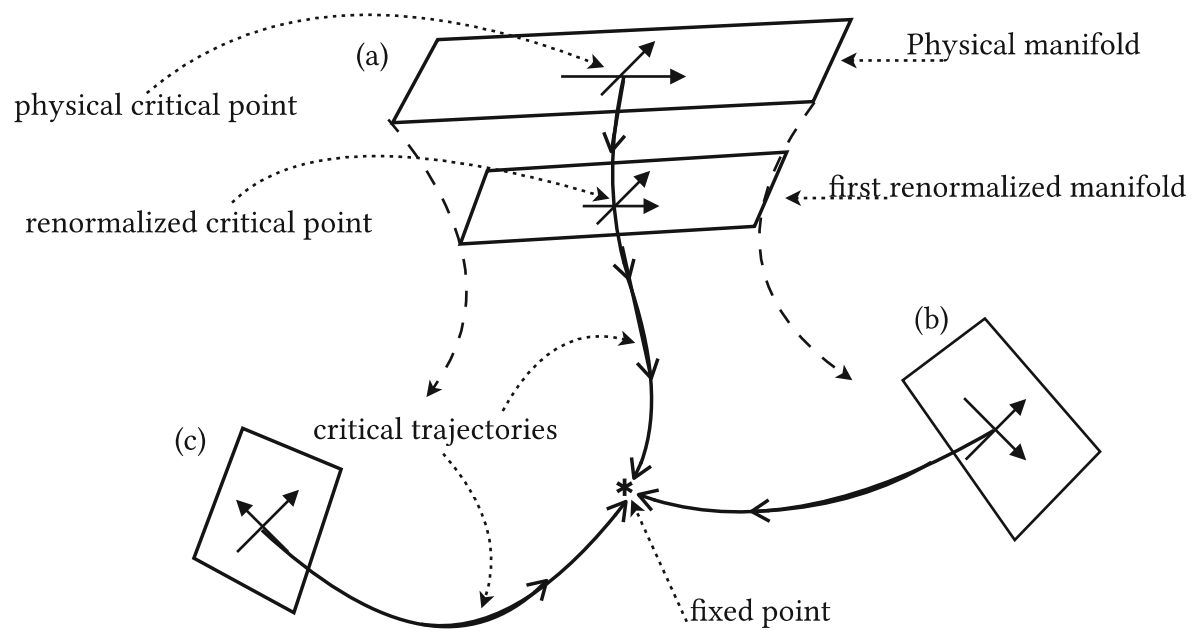

Fig. 2 Flow of Hamiltonians. a, b, and $\mathbf{c}$ represent different physical manifolds. Each manifold has a critical trajectory toward the same fixed point *

length is presumed. ${ }^{15}$ Once the fixed point is found, the system can be characterized by critical exponents and its universality class. Importantly, the fixed points are a product of the transformations, and with each transformation, the behavior of the system is still represented, albeit at a larger length scale.

Now to the main question of this paper: Are RG explanations of universality causal or noncausal? In what follows I first consider what reasons are given for a noncausal interpretation, closely considering Batterman (2000, 2018) and Reutlinger's (2014) interpretation, before arguing that these reasons are under-supported. As will be made clear, part of the problem rests on the inherent ambiguity in the explanandum and what it is we lose and don't lose when we abstract away the microphysical details of the system and its short-range interactions.

\section{Noncausal interpretation}

Batterman (2000) originally argued that RG explanations of a single system's behavior provide us with a noncausal explanation simply because they abstract away low-level causal details. However, this general challenge to causal accounts of explanation has since been called into question. Nearly all accounts of causal explanation make room for causal abstraction. For example, Strevens (2008) and Woodward's (2010) approaches to explanation make room for causal abstraction by utilizing concepts such as difference-making and causal specificity, respectively. When one abstracts away causal particularities that are not necessary for the entailment of the explanandum, one is still left with a causal explanation. It is an explanation that focuses on the causal influences - using Strevens' language — that made a difference. For example, leaving

\footnotetext{
15 The reliance on an infinite correlation length at the critical point may have interesting philosophical implications concerning idealization (Rice 2018) and whether RG explanations are reductive (Saatsi and Reutlinger 2018).
} 
out particle interactions when explaining gaseous behavior with the ideal gas law can still leave us with a causal explanation, despite leaving out such detail.

Reutlinger $(2014,2016)$ takes a different approach. He readily admits that a system's Hamiltonian gives us causal information. It is an energy function that maps the interactions between the degrees of freedom, spins, the effects of external fields, and so on. He argues that when we move to the transformations of Hamiltonians that exploit mathematical operations, one cannot appeal to accounts of causal explanation that make room for causal abstraction. He says the following:

Suppose Hamiltonians $H$ and $H^{*}$ represent the interactions of the microcomponents of some fluid $F$, and $H^{*}$ is the product of an RG transformation on the "real physical" $H$. If this is the situation, then it is not the case that Hamiltonian $\mathrm{H}$ occurs before (or after) Hamiltonian $H^{*}$, because Hamiltonians simply do not stand in temporal relations. It also seems to be inaccurate to say that $H$ and $H^{*}$ stand in any significantly asymmetric dependence relation because $\mathrm{H}$ and $H^{*}$ are equivalent representations of the same fluid $F$ (when undergoing phase transition). Therefore, neither of the two mathematical operations involved in $\mathrm{RG}$ explanations is best understood as directly revealing information about cause-effect relations. (2014, 1168-9)

The argument here first suggests that the explanatory power comes in part from the coarse graining process. And since different energy functions (Hamiltonians) are not themselves standing in a temporal relationship to each other, nor are they themselves events, we have a candidate for noncausal explanation, since two of the main common sense notions of causation (time-asymmetry and event tokens) are not present. The case becomes stronger when we compare disparate systems, moving to an inter-system explanation of universality. Unlike the single system RG explanation, an inter-system RG explanation explains why there are macrolevel similarities between disparate systems. It is even less clear if abstracting away irrelevant causal details in this manner still leaves us with a causal explanation. It seems that when we explain similarities between systems we are not only abstracting away from irrelevant causal details of the specific systems, but we are ignoring causal details altogether.

Thus, Reutlinger concludes that, on his counterfactual theory of explanation, the following counterfactual, underlying RG explanations of universality (which I am calling RGC), is noncausal:

RGC: There is a physical possible Hamiltonian $\mathrm{H}^{*}$ such that if (1) a physical system had the original Hamiltonian $\mathrm{H}^{*}$ (instead of its actual original Hamiltonian $\mathrm{H}$ ), (2) $\mathrm{H}^{*}$ were subject to repeated RG transformations, and (3) we determined the resulting flow of the Hamiltonians to a fixed point, then a system with original $\mathrm{H}^{*}$ would be in a different universality class than a system with original Hamiltonian H. $(2016,743)$

The claim is that the causal-tether that the original Hamiltonian had is no longer relevant; the causal background of each system's respective Hamiltonian is presupposed. The underlying causes of each system are abstracted away completely, 
leaving us to only compare the products of mathematical derivation or the abstract space of models. ${ }^{16}$ The mathematical procedures paramount to RG relate entire energy functions (Hamiltonians) to the space of possible energy functions, which are not the kind of thing that provides causal information. The RG transformations on the Hamiltonians in question do not have a time-asymmetric relationship. The mapping of two respective systems' transformed Hamiltonians is not an event or event-type. And yet, it is argued, it is precisely both these operations that primarily explain why disparate systems display similar macrobehavior at the critical point.

If this description is correct, then it is seems as though RG satisfies $\mathrm{NC}^{* *}$. When explaining why disparate systems display similar macrobehavior when undergoing a continuous phase transition, and thus share a universality class, we have a noncausal element that primarily explains the explanandum in question without a causal-tether. However, upon closer look at the role and levels of abstraction, I argue that this noncausal interpretation is under-motivated and does not preclude a causal interpretation.

\section{Noncausal interpretation challenged}

There are three central motivating reasons that noncausalists employ to suggest that there is no causal-tether in RG transformations and explanations of universality: 1) that we are abstracting away irrelevant causal details, 2) that there is no time-asymmetry or event types in RG transformations (i.e. our common sense notion of causation is not upheld), 3) that the explanatory power of universality is rooted in the abstract space of models, not in a causal mapping. It is my contention that none of these reasons actually support a noncausal interpretation. Thus, in order for noncausalists to continue to hold up RG as a classic example of noncausal explanation, more argumentation is needed. Let's take a closer look at each in turn.

\subsection{Ignoring causal details}

As mentioned above, Batterman (2000) originally argued that RG explanations of universality are noncausal simply because they abstract away lower-level causal details. It is worth considering this line of thought in more detail. Consider a more commonplace example: explaining why the glass broke with the ideal gas law. The ideal gas law can be part of a causal explanation of the glass breaking even if it ignores low-level particle interactions, and even though it is expressed mathematically. Moreover, one can also describe the same event using what Strevens (2008) calls the canonical explanation: the explanation that involves all the microlevel causal details. The important difference for our purposes is that a causal abstraction has taken place, where one

\footnotetext{
${ }^{16}$ The main point here is not that RG methods of explaining the universality of critical phenomena are not applicable to event explanation, but that the principle way of explaining the event does not represent causal information. The argument is that the primary reason that a RG explanation succeeds is because of the mathematical operations employed on what does not represent a causal relation. Indeed, in order for RG explanations to be an objection to causal accounts of explanation, there needs to be the implicit assumption that an event is being explained. In this case, it is an event of a continuous phase transition of several diverse systems sharing a universality class.
} 
explanation focuses on the low-level causal influences, while the other focuses just on the causal-difference makers. Many believe that both of these explanations are causal explanations despite the differing level of explanation. Moreover, both are causal even though one ignores low-level causal details. In order to show that an explanation is noncausal, noncausalists need to go beyond pointing out that low-level causal details were abstracted away; they need to show that a standard account of causal abstraction is not available for the case at hand.

However, noncausalists have not sufficiently ruled out this type of causal abstraction in the case of RG. As already stipulated by noncausalists, a system's Hamiltonian expresses causal information in mathematical language. It is an energy function that maps the interactions between the degrees of freedom, spins, the effects of external fields, and so on. Even though it is a mathematical representation, it falls within our commonsense notion of causation described at the outset. It represents interactions between the degrees of freedom, spins, the effects of external fields and other aspects of the system. When a system is undergoing a continuous phase transition, an event is occurring: the temperature gradually increases (or decreases), and there is a timeasymmetric relation between temperature increases and other components of the system. If we combine this observation with an approach that maintains timeasymmetry at the fundamental level (e.g. Ladyman and Ross's (2007) arrow of entropy), it makes the case that Hamiltonians convey time-asymmetric causal information even stronger. Moreover, just as with the ideal gas law, the same causal information can be represented at varying levels in the RG case. The transformed Hamiltonian at each step in the RG transformations still tracks the same various interactions among constituent particles in a system. The difference is that at each transformation, the Hamiltonian takes on a coarser-grained look at the system.

So, while the representation of the system changes through each transformation of the Hamiltonian, it does not thereby mean that the representation changes regarding its causal-tether. The transformation of the Hamiltonian does not express a change in the physical system, it expresses an alternative representation of the same process at the critical point. Once it is clear that a starting Hamiltonian, which Reutlinger (2014) admits, has a causal-tether, it is perfectly consistent to say that the transformed representations retain a causal-tether mapping of the interactions between the degrees of freedom, spins, the effects of external fields, and so on. One can simply apply whatever pet theory of causal abstraction she wishes to maintain that the transformed Hamiltonian has the same causal-tether. As an illustration, utilizing Strevens' (2008) account of causal abstraction, the abstraction process shows that short-range particle interactions are irrelevant to the macrobehavior of the system, so each transformation of the Hamiltonian isolates higher-level causal difference makers. On this view, we have an abstract yet still causal explanation of the critical phenomena of a single system.

Instead of RG explanations providing information regarding causal alternatives or a range of alternatives to a phase transition event - a common feature of causal explanation - they provide an alternative causal representation of the same event with the same causal history. It is a representation that ignores details in such a way that it preserves the phenomenon while leading to tractability. As Saatsi and Reutlinger say, the RG "flows do not correspond to any physical change of the system, but rather capture equivalence classes of models that share the same long-distance physics" $(2018,470)$. The focus is on a representation that ignores the short-range fluctuations 
and exhibits long-range fluctuations instead. Simply focusing on the long-distance physics does not preclude a causal interpretation. Thus, just as in the case of explaining with the ideal gas law, in order to show that an RG explanation of a single system is not compatible with a causal interpretation, one needs to go beyond saying that some causal details are abstracted away.

That said, even if the explanation of a single system's critical behavior is compatible with a causal interpretation, it does not mean that explanations of inter-systems, or universality, is also causal. The steps taken to find the universality class represent another level of abstracting away causal irrelevancies. Through RG, we see which system-specific differences do not make a difference to certain kinds of macrobehavior and which ones do. RG methods allow us to see that diverse systems flow to the same fixed point, which enable the characterization of a shared universality class. The numerical value of critical exponents, a function of long-range fluctuations, is the same between disparate systems. The inter-system explanation highlights which features across distinct systems make a difference to the long-range behavior during continuous phase transition. We find that features of each system's spatial dimension and spin dimensionality contribute to that system belonging to one universality class or another. RG transformations of the Hamiltonians of two systems which belong to different universality classes would not reach the same fixed point or have the same critical exponents.

The difference between the single system explanation and the inter-system explanation is that in the inter-system explanation we abstract away the causal details specific to each system to reveal that the most general dependencies are spatial dimension, spin dimensionality, etc. which in part determines the universality class. The novel aspect of inter-system RG explanations is the macrolevel abstraction that explains and describes shared behavior and universality.

The question is whether just in virtue of abstracting away from some system-specific causal details is enough to conclude that the resulting explanation is noncausal. However, this doesn't seem to be the case. As with the case of the single-system explanation, there is nothing in principle that is special about an abstraction that crosses over several systems that precludes a causal interpretation. In the biological sciences, explanations in convergent evolution explain why disparate populations develop similar traits. For example, golden moles have several of the same phenotypes as marsupial moles despite not being evolutionarily related. They have similar fur and claws, lack sight, and overall look quite similar. ${ }^{17}$ Explaining why convergent evolution occurs such that diverse populations share the same phenotypes also abstracts away the particular causal history of a particular population. Yet the inter-system explanation highlights causal features that both populations share in common that led to each belonging to a phenotypic universality class, such as having a similar habitat.

There are also examples in the physical sciences. Consider an adaption of Salmon's (2006) example of explaining why the helium filled balloon goes toward the front of the plane during acceleration and takeoff instead of toward the rear. If we want to explain not just that a helium filled balloon exhibits this behavior, but also explain why a hydrogen balloon (and any balloon filled with a gas lighter than air) would exhibit the same behavior, we have an inter-system explanation. The explanation would highlight

$\overline{{ }^{17} \text { This example is from Strevens }}$ (2017). 
how the features that these gasses have in common give rise to the same behavior in response to the pressure gradient present during takeoff. Importantly, abstracting away the specific causal details unique to each balloon does not thereby undermine the causal nature of the inter-system explanation. The inter-system explanation will still depend on the causal features that are present in the single-system causal explanation of Salmon's original case.

Once it is open for causalists to interpret a single-system RG explanation as a causal explanation, the move claiming that inter-system explanation is just another level of abstraction, not fundamentally different in kind, is readily available. Similarly to the convergent evolution case and the balloon case, the inter-system explanation exploits features shared among disparate systems that give rise to the same long-distance physics. One can again apply whatever pet theory of causal explanation one chooses to tell a story about why abstracting these low-level system-specific details retains a causal-tether. For example, RG fits into what Potochnik (2015) calls a causal pattern, because RG highlights the properties of a system that the system depends on and the scope of that dependence. Abstracting away particular causal details between systems, and providing an inter-system explanation, does not by itself preclude a causal interpretation.

A noncausalist could object that the examples I gave of causal inter-system explanation are not actually cases of causal explanation. However, in that case, the noncausalist is arguing for a much stronger claim. It is not simply that RG is noncausal, but that other seemingly canonical cases of causal abstraction are themselves noncausal. Instead, a more promising route is for noncausalists to provide a further reason besides abstracting away low-level causal details to make their case.

\subsection{RG transformations do not represent causal relations}

One reason to think that RG transformations are fundamentally different from standard causal abstractions touted by causalists is the fact that the transformation and mapping of Hamiltonians are central to explaining universality. Unlike explanations that use an abstract causal representation at the start to explain a phenomenon of interest (e.g. an explanation utilizing the ideal gas law), a central component of RG explanations of universality is tracking the abstraction process itself. Reutlinger (2014, 1168-9) stresses that the RG transformations on the Hamiltonians in question primarily explain universality, yet these transformations are not the sort of thing that could be an event and "simply do not stand in temporal relations," let alone display temporal-asymmetry. Thus, it seems that $\mathrm{NC}^{* *}$ is satisfied: there is a noncausal element - the transformations of Hamiltonians - that primarily explains universality while lacking a causal-tether.

However, even though there is no temporality or time-asymmetry in the transformations of Hamiltonians, it still does not undermine a possible causal interpretation. Such a response fails to appreciate the role that abstraction plays. Any process of abstraction is not an event, if we disregard the model or the scientist performing the abstraction in sequential steps, nor is it the kind of thing that stands in a temporal relation displaying temporal-asymmetry between a higher or lower level. It is just what it is: an abstraction. Yet after the process of abstraction is complete, the more abstract representation can still hold on to the causal mapping of the less abstract version. When we are concerned about whether an explanation is causal, as discussed in section 2, we 
are diagnosing whether or not the abstraction continues to have a causal-tether; we are not merely explaining the mathematical relationship between two possible representations. Thus, pointing out that the relationship between two representations is not an event or does not connote time-asymmetry, as Reutlinger (2014) does, is a noncausalist's red-herring. It does not speak to the causal nature of the explanation.

Part of the problem here is that there is ambiguity in the explanandum, and several theorists are interested in slightly different explananda. ${ }^{18}$ When we ask the question, "why do diverse systems display the same macrobehavior during a continuous phase transition?" we could be interested in a number of different things. For instance, we might just be interested in the definition of a universality class or interested in the mathematics behind Hamiltonian flow and calculating critical exponents, that is, how the mathematical derivation behind the process of abstraction itself works. The relevant explananda for our purposes are ones that present a pressing challenge to causal-only approaches to scientific explanation.

A question that just seeks a definition as its answer is not a threat to a causal account (if it can even constitute an explanation at all). Furthermore, a question that simply seeks the mathematical derivation as an answer is not by itself a threat. The threat comes when the principal way of explaining an event relies on a relation other than a causal one. An explanation that is simply comparative or points only to a mathematical derivation leaves out a crucial explanatory step. How is it that the derivation speaks to the behavior of systems at the critical point? The explanatory power does not simply come from the fact that the original Hamiltonian equation can be manipulated mathematically, but that after going through an RG transformation it still tracks the behavior of systems at the critical point.

However, Reutlinger (2014) draws our attention just to the process of abstraction (i.e. the RG transformations) to make his noncausal argument, neglecting its connection to real physical systems. Furthermore, building off of Reutlinger (2014), Saatsi and Reutlinger $(2018,464)$ explicitly discuss the explanandum of interest as explaining how it follows "from the laws of statistical mechanics, including the dynamical laws and the partition function connecting the micro- and macrolevels, that the properties exhibiting universality" depend on the system's spatial dimensions and other features that determine universality. This explanandum more explicitly asks us for a mathematical derivation as a central part of the explanation, which again unduly puts the finger on the scale toward a noncausal interpretation. While Saatsi and Reutlinger are correct that the transformations of the Hamiltonians play a primary role in the explanation of universality, if the focus is only on the mathematical process behind these transformations, we lose the connection to the physical phenomenon. Franklin (2018) also argues, in a context other than the causal / noncausal debate, that any abstract explanation of universality that does not maintain the connection to the physical phenomenon by also explaining why systems A-E have certain behavior in common or why the differences between A-E are irrelevant to their behavior, falls short of providing the explanatory scope and answers to what-if-things-had-been-different

\footnotetext{
${ }^{18}$ See Franklin (2018) for a compatible discussion on ambiguities present in the literature concerning RG explananda, and specifically on the difference between simply comparing two RG explanations and giving a more unified explanation of universality.
} 
questions that we are essentially interested in when explaining the universality of continuous phase transitions. ${ }^{19}$

The important question in the causal debate for explaining why diverse systems display the same macrobehavior during a continuous phase transition is whether the transformed Hamiltonian still retains its causal tether to the real physical phenomenon. As I have argued above, the transformed Hamiltonian indeed still has this causal-tether. Specifically, unlike the strawberry case, RG methods in the case of critical phenomena are not divorced from the physics. If the laws of physics were different, the same explanation would not hold. There is something about how certain fluids are constituted that determines the system's universality class. For instance, there are special cases where substances undergo a continuous phase transition that otherwise wouldn't, ${ }^{20}$ and cases where substances engage in crossover phenomena where the system essentially changes its universality class in the process. ${ }^{21}$ Moreover, as discussed in the previous section, there is a time-asymmetric relation between temperature increases and other components of a system undergoing a continuous phase transition that the Hamiltonian represents. All this suggests that the temporal-asymmetry requirements to uphold our common sense intuitions about causes can be satisfied. ${ }^{22}$ Thus, simply pointing to the fact that the explanation heavily relies on abstraction procedures, such as RG transformations and Hamiltonian flow, does not preclude a causal interpretation.

\subsection{The abstract model space}

This brings us to the third and arguably the central reason noncausalists argue that RG explanations do not retain any causal-tether: the explanatory power in explaining universality is rooted in the abstract space of models (Hamiltonian flow), not in counterfactual causal reasoning or reasoning about causal difference-makers. According to Saatsi and Reutlinger (2018), considering the abstract space of Hamiltonian flow is what makes the explanation go beyond simple derivation. Batterman (2018), adapting his earlier views on abstracting away low-level causal details, argues that the topology of the space of RG flows - the combination of different possible models - is what explains the irrelevance of the short-range particle interactions, rather than causal features of individual systems. Unlike the reasoning discussed in the previous section, that mathematical abstraction procedures (i.e. the transformations of the Hamiltonians) undermine a causal interpretation, the concern here is that the topology of the space of RG flows noncausally explains universality. Questions of

\footnotetext{
${ }^{19}$ Saatsi and Reutlinger (2018) do seek to go beyond derivation for explaining universality. They do so by appealing to the topology of the abstract model space, which I discuss in section 5.3.

${ }^{20}$ See footnote 7.

${ }^{21}$ See Franklin $(2018,241)$ for a discussion of crossover in the context of explanation and universality.

22 One could argue that the temporal asymmetry that matters here is not that the Hamiltonian representation conveys time-asymmetry, but that the relationship between the explanans and the explanandum itself stands in a time-asymmetric relation. However, this response is underdeveloped. Which aspects of the explanantia must be temporally prior? As discussed in section 2, causally explaining why all ravens are black includes facts along the lines, "all ravens have some property $p$." Yet, having a particular property could be temporally continuous with blackness. Instead, if we adopt the counterfactual account of explanation, what matters is that the explanation is explanatory by virtue of exhibiting an asymmetrical counterfactual dependency. This counterfactual could be causal or noncausal. Thus, the question here is whether RGC is a causal counterfactual. I consider this question specifically in section 5.3.
} 
time-asymmetry again emerge. The topology of an abstract space of models is not something that has a temporal character, and thus does not represent a time-asymmetric dependence relation.

Here's the problem: the fact that RG explanations exploit and consider the space of abstract models - all the varying causal representations (Hamiltonians) that flow toward a fixed point - does not alone speak for or against the causal nature of the explanation. Consider a different example where an explanation relies on comparisons between models. The United States National Weather service and climate scientists utilize several different models in order to get better predictions, explore tradeoffs, and explore causal influences (Wiesberg 2007). Instead of one model, there is a set of models that each highlight different factors that together account for all the core causal factors. Importantly, the practice of considering these multiple models is still chiefly concerned with the causal-tether the set of models has to the phenomenon, not simply the formal relationship between the models. Thus, it is not simply because an explanation is rooted in the space of abstract models that the explanation lacks causal-tether.

The unmotivated assumption here is that in the case of RG transformations on Hamiltonians, considering the set of models (the set of all the transformed Hamiltonians) lacks a causal-tether. However, since noncausalists have not ruled out that each particular transformed Hamiltonian has a causal-tether, and they have not ruled out that the abstract space of models gives us a series of alternative causal representations of the same event, then considering the abstract space of these models can plausibly be taken to be similar to considering causal tradeoffs and influences in the multiple model case. And again, the claim that key causal, such as time-asymmetry, are lacking in considering the abstract model space is a red-herring. The burden of proof is on showing why comparing a set of models of the same event, where each model has a causal-tether, cannot causally explain. Unfortunately, no such argument is given.

Putting all this together, consider again RGC:

RGC: There is a physical possible Hamiltonian $\mathrm{H}^{*}$ such that if (1) a physical system had the original Hamiltonian $\mathrm{H}^{*}$ (instead of its actual original Hamiltonian $\mathrm{H}$ ), (2) $\mathrm{H}^{*}$ were subject to repeated RG transformations, and (3) we determined the resulting flow of the Hamiltonians to a fixed point, then a system with original $\mathrm{H}^{*}$ would be in a different universality class than a system with original Hamiltonian $\mathrm{H}$.

Recall that Reutlinger (2016) argues that RGC is noncausal, thereby making the primary locus of explanatory power a noncausal dependency relation, thereby satisfying $\mathrm{NC}^{* *}$. However, it should be clear at this point that this claim is not supported. First, a system's physical Hamiltonian is grounded in causal features. Like in the billiard ball case - there is something about the constitution of the billiard balls that determines that they will behave a certain way under certain conditions - there is something about the constitution of substances that determines how they behave during a continuous phase transition and determines their universality class. As discussed in section 3, a system's order parameter, which is fundamental to determining universality class, is only known through empirically observing the system and how it behaves under various conditions. This is altogether different from the strawberry case where there is nothing about the 
strawberries in particular that prevents them from being divided in the way described. Second, the causalist can maintain through any standard conception of causal abstraction that the RG transformations of the physical Hamiltonian are simply alternative representations of the same information, albeit pointing out more macrolevel causal details. So, while the process of transformation is not a causal process, the representation that remains after this process still holds on to our common sense notions of causation. The new Hamiltonian tracks the long-distance physics of an event that conveys temporal-asymmetry and metaphysical contingency. While tracing the system to a fixed point again is not a causal process - it is a method used to uncover and categorize commonalities - it is still consistent with a causal interpretation that explaining can involve diverse methods and still retain a causal-tether to the phenomenon.

In the end, the now widely held assumption that RG explanations of universality are noncausal has not been well supported. The reasons given for why the mathematical representations and operations behind RG explanations lack a causal-tether are undermotivated. The main stumbling block seems to be that a system's original Hamiltonian provides us with causal information in mathematical language, in a similar way as the collision of two billiard balls can be encapsulated in mathematical language. This initial causal-tether enables causal accounts of explanation to supply causal interpretations even as layers of abstraction are added, and even when the abstraction is across systems.

\section{Conclusion}

Renormalization group explanations of the universality of critical phenomena are held up as a canonical case of noncausal explanation. However, the arguments given for why it is an instance of noncausal explanation are not very compelling. In this paper, I argued that for all that noncausalists have said about why RG explanations are noncausal, a causal interpretation is still available. Moreover, I have done this using a fairly demanding view of causation that noncausalists themselves endorse, where the nature of causes is bound to common sense notions concerning time-asymmetry, eventtypes, and metaphysical contingency.

While there may be other reasons why RG explanations are noncausal, these reasons have not been explicitly explored thus far in the literature. As a result, proponents of noncausal accounts need to expand their toolbox. Moreover, if the primary case held up by pluralists employing the inter-system irrelevancy approach to noncausal explanation fails to withstand this kind of scrutiny, then the lines between causal explanation and noncausal explanation demands closer attention, as does the question whether cases stemming from mathematical necessity, like the strawberry case, are also affected.

Acknowledgements For many helpful comments and conversations I would like to thank the anonymous referees, Nathan Ballantyne, Stephen Grimm, Diana Heney, Kareem Khalifa, Insa Lawler, Juha Saatsi, Peter Seipel, Michael Strevens, Coran Stewart, with special thanks to John Mumm. I presented this work at Fordham University and at CUNY Graduate Center as a part of SWIP-Analytic. I thank the attendees for the very fruitful discussions and suggestions that followed. 
Open Access This article is distributed under the terms of the Creative Commons Attribution 4.0 International License (http://creativecommons.org/licenses/by/4.0/), which permits unrestricted use, distribution, and reproduction in any medium, provided you give appropriate credit to the original author(s) and the source, provide a link to the Creative Commons license, and indicate if changes were made.

\section{References}

Albert, D. (2000). Time and chance. Cambridge, MA: Harvard University Press.

Batterman, R. (2000). Multiple Realizability and universality. The British Journal for the Philosophy of Science, 51, 115-145.

Batterman, R. (2010). On the explanatory role of mathematics in empirical science. The British Journal for the Philosophy of Science, 61, 1-25.

Batterman, R. (2018) Autonomy of theories: An explanatory problem. Noûs, 52(4), 858-873.

Binney, J. J., Dowrick, N. J., Fisher, A. J., \& Newman, M. (1992). The theory of critical phenomena: An introduction to the renormalization group. Oxford: Oxford University Press, Inc.

Blanchard, T. (2016). Physics and causation. Philosophy Compass, 11(5), 256-266.

Butterfield, J. (2011). Less is different: Emergence and reduction reconciled. Foundations of Physics, 41, $1065-1135$.

Butterfield, J. (2014). Reduction, emergence, and renormalization. The Journal of Philosophy, 111, -15, 49.

Butterfield, J., \& Bouatta, N. (2012). Emergence and reduction combined in phase transitions. Frontiers of Fundamental Physics, 1446, 383-403.

Colyvan, M. (2001). The indispensability of mathematics. New York: Oxford University Press.

Farr, M., \& Reutlinger, A. (2013). A relic of a bygone age? Causation, time symmetry and the directionality argument. Erkenntnis, 78(2), 215-235.

Felline, L. (2018). Mechanisms meet structural explanation. Synthese, 195(1), 99-114.

Franklin, A. (2018). On the renormalization group explanation of universality. Philosophy of Science, 85(2), 225-248.

Jansson, L., \& Saatsi, J. (2017). Explanatory abstractions. The British Journal for the Philosophy of Science. https://doi.org/10.1093/bjps/axx016.

Kadanoff, L. (1966). Scaling Laws for Ising models near $T_{c}$. Physics, 2, 263-272.

Kadanoff, L. (2013). Relating theories via renormalization. Studies in History and Philosophy of Modern Physics, 44, 22-39.

Khalifa, K., Doble, G., \& Millson, J. (2018). Counterfactuals and explanatory pluralism. British Journal for the Philosophy of Science. https://doi.org/10.1093/bjps/axy048.

Ladyman, J., \& Ross, D. (2007). Everything must go. Metaphysics naturalized. New York: Oxford University Press.

Lange, M. (2013). What makes a scientific explanation distinctively mathematical? British Journal for the Philosophy of Science, 64, 484-511.

Lange, M. (2017). Because without cause. Oxford: Oxford University Press.

Lipton, P. (2004). Inference to the best explanation. New York: Routledge.

Loewer, B. (2007). Counterfactuals and the second law. In H. Price \& R. Corry (Eds.), Causation, Physics, and the Constitution of Reality: Russell's Republic Revisited (pp. 293-326). Oxford: Clarendon Press.

Morrison, M. (2014). Complex systems and renormalization group explanations. Philosophy of Science, 81, 1144-1156.

Pincock, C. (2015). Abstract explanations in science. The British Journal for the Philosophy of Science, 66(4), $857-882$.

Potochnik, A. (2015). Causal patterns and adequate explanations. Philosophical Studies, 172(5), 1163-1182.

Povich, M. (2016). Minimal models and the generalized ontic conception of scientific explanation. The British Journal for the Philosophy of Science, 69(1), 117-137.

Reutlinger, A. (2014). Why is there universal macrobehavior? Renormalization group explanation as noncausal explanation. Philosophy of Science, 81, 1157-1170.

Reutlinger, A. (2016). Is there a monist theory of causal and noncausal explanations? The counterfactual theory of scientific explanation. Philosophy of Science, 83(5), 733-745.

Reutlinger, A. (2017). Explanation beyond causation? New directions in the philosophy of scientific explanation. Philosophy Compass, 12, 21-11.

Rice, C. (2015). Moving beyond causes: Optimality models and scientific explanation. Nous, 49, $589-615$.

Rice, C. (2018). Idealized models, holistic distortions, and universality. Synthese, 195(6), 2795-2819. 
Russell, B. (1913). On the notion of cause. Proceedings of the Aristotelian Society, 13, 1-26.

Saatsi, J., \& Pexton, M. (2013). Reassessing Woodward's account of explanation: Regularities, counterfactuals, and noncausal explanations. Philosophy of Science, 80(5), 613-624.

Saatsi, J., \& Reutlinger, A. (2018). Taking reductionism to the limit: How to rebut the antireductionist argument from infinite limits. Philosophy of Science, 85(3), 455-482.

Salmon, W. C. (2006). Four decades of scientific explanation. Pittsburgh: University of Pittsburgh Press (Original work published 1989).

Skow, B. (2014). Are there non-causal explanations (of particular events)? The British Journal for the Philosophy of Science, 65(3), 445-467.

Skow, B. (2016). Reasons why. Oxford: Oxford University Press.

Strevens, M. (2008). Depth. Cambridge, MA: Harvard University Press.

Strevens, M. (2017). The whole story. In D. Kaplan (Ed.), Explanation and Integration in Mind and Brain Science. Oxford: OUP.

Wiesberg, M. (2007). Three kinds of idealization. The Journal of Philosophy, 104(12), 639-659.

Woodward, J. (2003). Making Things Happen. New York: Oxford University Press.

Woodward, J. (2010). Causation in biology stability, specificity, and the choice of levels of explanation. Biology and Philosophy, 25, 287-318.

Publisher's note Springer Nature remains neutral with regard to jurisdictional claims in published maps and institutional affiliations. 